\title{
Efecto del extracto acuoso de maca sobre la función cognitiva en ratas recién destetadas
}

Effect of aqueous extract of maca on cognitive function in weaning infant rats

\author{
Raquel Oré ${ }^{1}$, Silvia Suárez ${ }^{1}$, Luis Rojas², Rubén Valdivieso', Rosa Oriondo, \\ Fernando Tapia ${ }^{3}$, Juan Trabuco ${ }^{3}$ \\ ${ }^{1}$ Centro de Investigación de Bioquímica y Nutrición. Facultad de Medicina, Universidad Nacional Mayor de San Marcos. Lima, Perú. \\ ${ }^{2}$ Facultad de Farmacia y Bioquímica, Universidad Nacional Mayor de San Marcos Lima, Perú. \\ ${ }^{3}$ Facultad de Medicina, Universidad Nacional Mayor de San Marcos Lima, Perú. Tesistas de la Maestría en Bioquímica.
}

\begin{abstract}
Resumen
Introducción: La maca, Lepidium meyenii walp, es una planta que crece en las alturas de los andes peruanos; es usada como alimento, por su valor nutricional y sus propiedades etnomedicinales, unidos a la fertilidad y vitalidad. En la actualidad, se ha incrementado el interés público en los polifenoles, que tendrían efecto inhibitorio sobre la actividad acetilcolinesterasa y butirilcolinesterasas, y de esta manera se mejoraría las funciones cognitivas, aunque los mecanismos aún no han sido dilucidados. Por esta razón, se ha propuesto que la maca es un adaptógeno, que aumenta la energía, resistencia y reduce el estrés. Objetivos: Demostrar y evaluar el efecto energizante de la maca amarilla sobre la memoria y el aprendizaje por medio de pruebas bioquímicas, en un modelo animal de ratas destetadas. Diseño: Estudio experimental. Institución: Centro de Investigación de Bioquímica y Nutrición. Facultad de Medicina, Universidad Nacional Mayor de San Marcos. Lima, Perú. Material biológico: Ratas recién destetadas y extracto acuoso de maca amarillo. Intervenciones: A ratas recién destetadas se les administró extracto acuoso de maca amarillo (0,4 mg, 0,8 mg y 1,2 mg/g de peso), durante 15 dias, y luego se les sometió a la prueba de aprendizaje espacial acuática de Morris. También, se tomó muestras de cerebro para determinaciones bioquímicas. Principales medidas de resultados: Mejoria del aprendizaje y la memoria. Resultados: Se encontró inhibición de la butirilcolinesterasa, cuando se administró dosis crecientes de maca, excepto el grupo que recibió 0,8 mg/g. Los niveles de lipoperoxidación en el cerebro (daño oxidativo) disminuyeron al incrementar la dosis de maca; así mismo, los tiempos (s) en hallar la plataforma en la prueba de Morris disminuyeron al incrementar la dosis de maca amarilla. La concentración de glutatión en el cerebro se incrementó con la administración de maca, pero sin ser significativo. Conclusiones: La administración de extracto acuoso de maca mejora el aprendizaje y la memoria en animales de experimentación, así como las defensas antioxidantes en cerebro.
\end{abstract}

Palabras clave: Maca amarilla, ratas, memoria, aprendizaje, glutatión, lipoperoxidación, butirilcolinesterasa, prueba espacial acuática de Morris.

\begin{abstract}
Background: Maca, Lepidium meyenii walp, is a plant that grows in Peruvian Andes' highlands. It is used as food because of its nutritional value and its ethnical and medical properties related to fertility and vitality. Currently public interest has increased on food and dietetic supplements that contain active principles like polyphenol compounds with inhibitory effect on acetylcholinesterase and butyrylcholinesterase activities, thus improving cognitive functions through mechanisms yet unknown; for this reason maca has been proposed as an adaptogen that increases energy and resistance and diminishes stress. Objectives: To determine the energetic effect of yellow maca on memory and learning by biochemical tests, in an animal model of weaning rats. Design: Experimental study. Setting: Biochemistry and Nutrition Research Center, Faculty of Medicine, Universidad Nacional Mayor de San Marcos, Lima, Peru. Biologic material: Weaning rats and aqueous yellow maca extract. Interventions: Weaning rats received yellow maca liquid extract $(0.4 \mathrm{mg}, 0.8$ $\mathrm{mg}$, and $1.2 \mathrm{mg} / \mathrm{g}$ de peso) during 15 days followed then by Morris spatial aquatic test. Brain samples were taken for biochemistry tests. Main outcome measures: Improvement of both learning and memory. Results: Butyrylcholinesterase inhibition was found with increasing dosis of maca except the group receiving $0.8 \mathrm{mg} / \mathrm{g}$. Brain lipoperoxidation level (oxidative damage) decreased with increasing maca dosis; also time ( $\mathrm{s}$ ) in finding Morris platform test decreased with increasing yellow maca dosis. Brain total glutation concentration increased not significatively with maca supply. Conclusions: Maca liquid extract supplement improved learning and memory in experimental animals as well as brain antioxidant defenses.
\end{abstract}

Key words: Yellow maca, rats, memory, learning, glutathione, lipoperoxidation, butyrylcholinesterase, Morris spatial aquatic test.

An Fac med. 2011;72(1):13-6

\section{INTRODUCCIÓN}

La maca, Lepidium meyenii walp, es una planta arbustiva bianual, perteneciente a la familia Brassicaceae, que crece principalmente en la zona central del Perú, a una altura de 3500 a $4500 \mathrm{msnm}$.
Es usada como alimento, por su valor nutricional y sus propiedades etnomedicinales unidos a la fertilidad y vitalidad. En la puna se encuentra la fuente mayor de variabilidad genética ${ }^{(1)}$.

En quechua es conocida como maca, maka, maino, ayak chichita, ayak willku, y, en inglés, como maca y Peruvian ginseng.

En la actualidad, se ha incrementado el interés público en los alimentos y suplementos dietéticos que contienen principios activos como compuestos polifenólicos ${ }^{(2)}$, que tendrían efecto in- 
hibitorio sobre la actividad de la acetilcolinesterasa y butirilcolinesterasa, y de esta manera podría mejorar las funciones cognitivas, aunque los mecanismos aún no han sido dilucidados. Los polifenoles son sustancias químicas encontradas en plantas y frutas, caracterizadas por la presencia de más de un grupo fenol por molécula. Estos representan más de 8000 diferentes compuestos y son separados en diferentes clases por su estructura química. Los polifenoles más abundantes en las plantas son los flavonoides y los ácidos fenólicos, que dan cuenta de $60 \%$ y $30 \%$, respectivamente, de los polifenoles presentes en la dieta. La capacidad antioxidante in vitro de los polifenoles ha quedado demostrada, puesto que se ha observado su actividad como agentes reductores, quelantes de metales y como captadores de radicales libres, los cuales han sido involucrados en diversas enfermedades, como las neurodegenerativas, diabetes, cáncer, ateroesclerosis, entre otras $^{(3,4)}$.

Estos alimentos nutracéuticos son considerados como alternativos o complementarios a los tratamientos médicos convencionales, tanto para la prevención como para el manejo de diferentes enfermedades. Por esta razón, se ha propuesto que la maca es un adaptógeno, aumenta la energía, resistencia y reduce el estrés.

En el presente trabajo se propone demostrar algunos de los mecanismos que explicarían estas propiedades de la maca, teniendo en cuenta que de su composición se conoce que 55 a $60 \%$ consiste en carbohidratos, 10 a $12 \%$ en proteínas, 8 a $9 \%$ en fibra y 2 a 3\% en lípidos; así también, contiene ácidos grasos (macaneo) y sus correspondientes amidas (macamidas), glucosinolatos, alcaloides (lepidilinas A y B, macaridina), esteroles, ácido (1R, 3S)-1-metiltetrahidro- $\beta$-carboxílico y polifenoles, además de aminoácidos esenciales, hierro, calcio y otros metabolitos ${ }^{(5-7)}$.

Nuestros objetivos fueron demostrar y evaluar el efecto energizante de la maca amarilla sobre la memoria y el

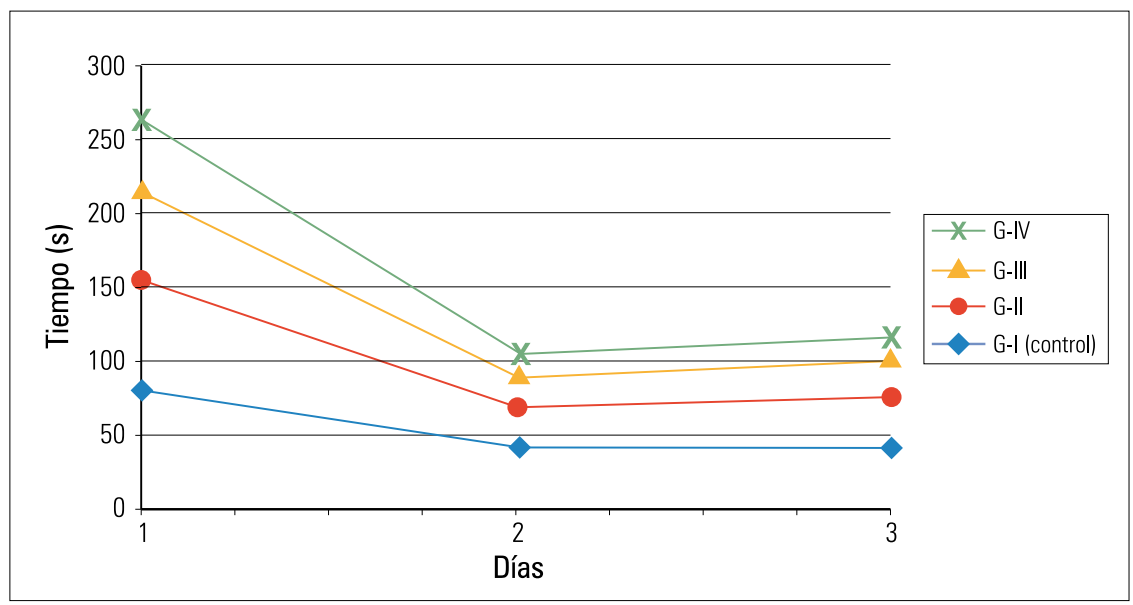

Figura 1. Tiempo (s) de aprendizaje en ratas recién destetadas tratadas con maca, según la prueba de Morris. G-I = grupo control. G-II = grupo al que se administró maca $0,4 \mathrm{mg} / \mathrm{g}$ de peso. G- III = grupo al que se dio maca $0,8 \mathrm{mg} / \mathrm{g}$ de peso. G-IV = grupo al que se administró maca $1,2 \mathrm{mg} / \mathrm{g}$ de peso. aprendizaje, mediante la prueba espacial acuática de Morris y pruebas bioquímicas, en un modelo animal de ratas destetadas.

\section{MÉTODOS}

Se utilizó ratas recién destetadas, a las cuales se les administró extracto acuoso de maca amarillo $(0,4 \mathrm{mg}, 0,8 \mathrm{mg}$ y 1,2 $\mathrm{mg} / \mathrm{g}$ de peso), vía orogástrica, durante 15 días, y luego fueron sometidas a la prueba de aprendizaje y memoria espacial acuática. Para la evaluación del aprendizaje, la prueba espacial acuática de Morris se realizó durante 3 días consecutivos. En un día, cada período de ensayo tenía una duración máxima de 2 minutos, seguido de 20 segundos de descanso; se la repetía consecutivamente 3 veces. Se registró el tiempo que cada animal necesitó para alcanzar la plataforma. Los animales debían nadar en la piscina hasta localizar la plataforma. La prueba de memoria se realizó el cuarto día. Los animales fueron sometidos a la misma prueba, pero sin la plataforma. Se midió los tiempos de permanencia en el cuadrante donde estuvo la plataforma. El grupo control no recibió tratamiento alguno y fue sometido a los mismos ensayos.

Después de realizadas ambas pruebas, los animales fueron sacrificados por decapitación y se tomó muestras de

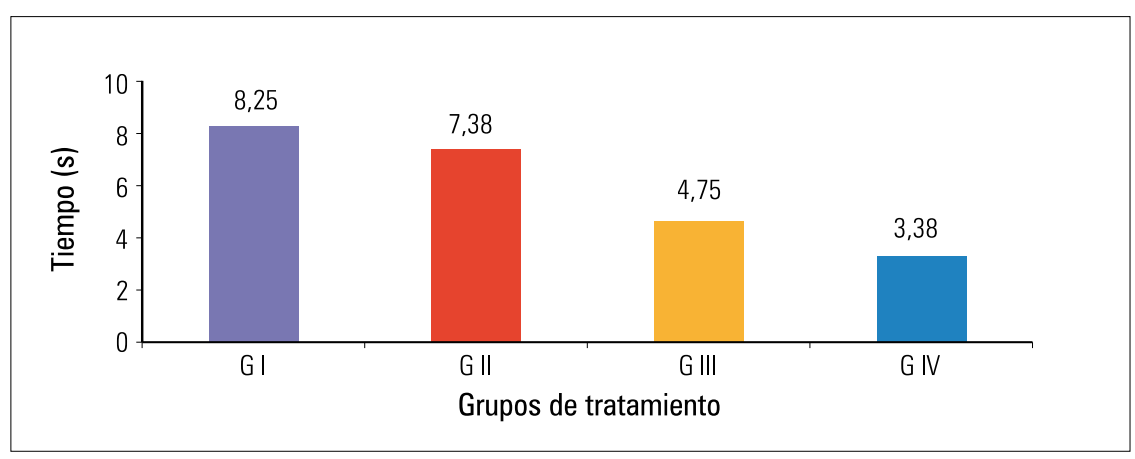

Figura 2. Tiempo en segundos (s) en la prueba de memoria en ratas destetadas tratadas con maca, según la prueba de Morris. 
Tabla 1. Parámetros de estrés oxidativo en homogenizado de cerebro de ratas tratadas con maca.

\begin{tabular}{|c|c|c|c|c|}
\cline { 2 - 5 } \multicolumn{1}{c|}{} & \multicolumn{4}{c|}{ Grupo de tratamiento } \\
& $\mathrm{G}-\mathrm{I}$ & $\mathrm{G}-\mathrm{II}$ & $\mathrm{G}-\mathrm{III}$ & $\mathrm{G}-\mathrm{IV}$ \\
& (control) & $0,4 \mathrm{mg} / \mathrm{g}$ & $0,8 \mathrm{mg} / \mathrm{g}$ & $1,2 \mathrm{mg} / \mathrm{g}$ \\
\hline \multirow{3}{*}{$\mathrm{MDA}$-TBARS $(\mu \mathrm{mol} / \mathrm{g})$} & $0,5654 \pm$ & $0,5538 \pm$ & $0,3923 \pm$ & $0,3731 \pm$ \\
& 0,0807 & 0,0692 & 0,0769 & 0,042 \\
& $\mathrm{a}, \mathrm{b}$ & $\mathrm{c}, \mathrm{d}$ & $\mathrm{a}, \mathrm{c}$ & $\mathrm{b}, \mathrm{d}$ \\
\hline \multirow{2}{*}{$\mathrm{GSH}(\mu \mathrm{mol} / \mathrm{g})$} & $0,322 \pm$ & $0,328 \pm$ & $0,2396 \pm$ & $0,34025 \pm$ \\
& 0,0211 & 0,0170 & 0,1793 & 0,0090 \\
\hline
\end{tabular}

cerebro para las determinaciones de la actividad enzimática de butirilcolinesterasa y los niveles del complejo MDATBARS y glutatión.

\section{RESULTADOS}

Al analizar los resultados sobre aprendizaje durante los tres días de la prueba, se observó diferencia significativa $(\mathrm{p}<0,05)$ de los grupos que recibieron maca, respecto al grupo control (figura 1).

El cuarto día, cuando se evalúa la memoria, los grupos III y IV (de mayor concentración de maca) mostraron mayor retención, reflejada en una reducción del tiempo en hallar el lugar donde se encontraba la plataforma, con respecto al control y al grupo II $(\mathrm{p}<$ 0,05) (figura 2).

Al evaluar marcadores de daño oxidativo en cerebro, se observó que la administración de maca disminuyó significativamente los niveles de lipoperoxidación en los grupos III y IV, respecto al grupo control y al grupo II. En el análisis del glutatión, se observó un incremento no significativo de esta molécula antioxidante en los grupos que recibieron maca (tabla 1 ).

Se investigó la actividad de la enzima butirilcoliesterasa en cerebro de rata, para evaluar la actividad cognitiva, observándose que los grupos que recibieron extracto de maca en las tres concentraciones produjo una disminución de los valores enzimáticos con respecto al grupo control (figura 3).
El interés médico y científico ha producido diversos modelos animales con el fin de comprender los mecanismos cerebrales que operan en el almacenamiento y la recuperación de la información.

La prueba espacial acuática de Morris es una prueba de gran utilidad para cuantificar la memoria, especialmente en combinación con técnicas electrofisiológicas. Es la más utilizada en animales de experimentación, para evaluar el comportamiento, especialmente el aprendizaje y la memoria.

En el presente estudio se ha aplicado la prueba de Morris en combinación con pruebas bioquímicas, que incluyó la enzima butirilcolinesterasa -relacionada con la memoria y aprendizaje- y metabolitos de estrés oxidativo propios del envejecimiento y de enfermedades neurodegenerativas, como herramientas para observar los efectos de la maca amarilla.

Hemos encontrado que la administración de maca amarilla a diversas concentraciones $(0,4 \mathrm{mg}, 0,8 \mathrm{mg}$ y 1,2 $\mathrm{mg} / \mathrm{g}$ de peso) mejora el aprendizaje, disminuyendo el tiempo de la prueba (segundos) al 3 día, en un rango de 23, 37 y $65 \%$, respectivamente, al compararlos con el grupo control. Respecto a la prueba para valorar la memoria, la administración de maca en diferentes concentraciones produjo disminución del tiempo para recordar la zona donde se hallaba la plataforma, después

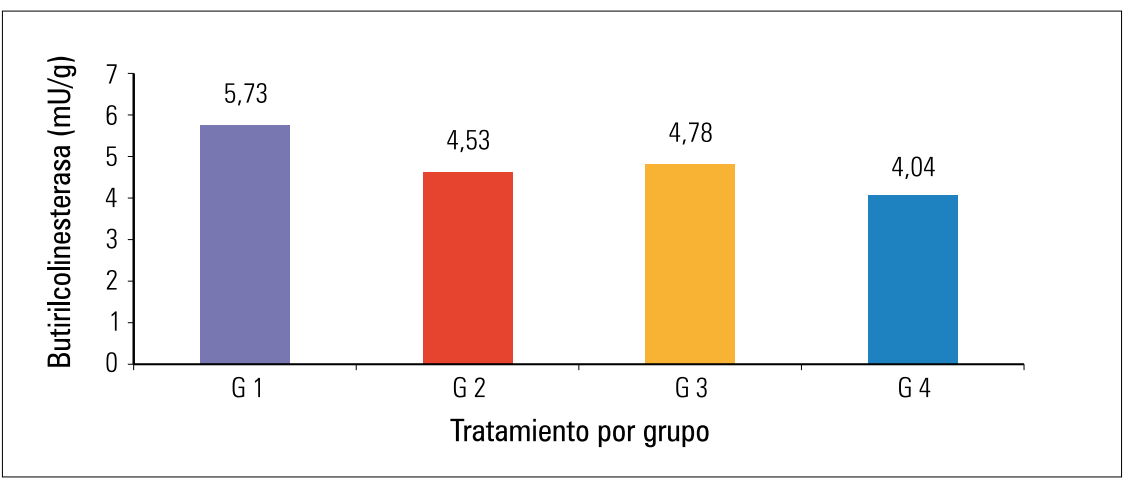

Figura 3. Actividad de la butirulicolinesterasa $(\mu \mathrm{m} / \mathrm{g})$ en el cerebro de las ratas destetadas, según tratamiento. Existe diferencia significativa entre los grupos: $\mathrm{G}_{1}-\mathrm{G} 4 ; \mathrm{G} 3-\mathrm{G} 4$ con $\mathrm{p}<0,05$. 
que fue retirada. Estudios realizados por Rubio y col. ${ }^{(8)}$ demostraron que la administración de maca negra mejora el daño causado por escolapina, que es una droga que favorece la pérdida de la memoria.

Investigaciones realizadas con una hierba china, en las que se aisló huperzine $\mathrm{A}$-un fármaco utilizado para el tratamiento de la enfermedad de Alzheimer-, hallaron que revierte o atenúa el déficit cognitivo en modelos de animales de experimentación, al inhibir la actividad de la enzima acetil colinesterasa ${ }^{(9,10)}$. Estudios realizados por Ezio Giacobini ${ }^{(11)}$ en pacientes con Alzheimer tratados con rivastigmina hallaron disminución de la actividad de la butirilcolinesterasa; esto indicaría una mejoría cognitiva. Nosotros evaluamos la actividad de la enzima butirilcolinesterasa en tejido cerebral, observándose reducción significativa de la actividad en los grupos que recibieron maca, al comparar los grupos III y IV; y el grupo IV frente al grupo control.

Estudiando los marcadores de estrés oxidativo, como el glutatión, hemos observado que la administración de maca incrementa el contenido total de este tripéptido antioxidante y reduce la formación del complejo MDA-TBARS, como protección del daño oxidativo. El glutatión es un metabolito endógeno que participa como antioxidante en varios sistemas biológicos, especialmente a nivel cerebral, y el compuesto derivado de la peroxidación lipídica malondialdehído (MDA) se produce en si- tuaciones fisiológicas y patológicas; una disminución de este proceso indica un efecto protector, especialmente a nivel de membranas.

Wang R y col. ${ }^{\left({ }^{9}\right)}$ mencionaron que el uso del fármaco huperzine $\mathrm{A}$ en el tratamiento de Alzheimer actuaría atenuando el daño oxidativo, regulando la expresión de proteínas apoptóticas Bcl-2, Bax, p53 y la caspasa-3, protegiendo a las mitocondrias.

De manera que, aquellos metabolitos que pueden proteger membranas también pueden participar, al menos de forma indirecta, en la regulación de la apoptosis.

Nuestros resultados permiten concluir que los metabolitos del extracto de maca amarilla actuán en diversos sistemas bioquímicos, disminuyendo la actividad de la enzima butirilcolinesterasa. Según la literatura, la actividad aumentada se relaciona con la pérdida de memoria y el aprendizaje. Igualmente, la administración de extracto de maca estaría actuando como un neuroprotector sobre los mecanismos de defensa antioxidantes.

\section{REFERENCIAS BIBLIOGRÁFICAS}

1. Gonzales GF. Biological effect of Lepidium meyenii, Maca, a plant from the highlands of Perú. En: Singh VK, Bardwaj R, Govil JN, Sharma RK (Eds). Recent Progress in Medical plants. Natural Products. USA: Studium Press LLC. 2006;15:217-42.

2. Oré MR. Efectos hipolipémico y antioxidante de Lepidium meyenii Walp en ratas. [Tesis Doctoral]. Lima: Sistema de Bibliotecas, Universidad Nacional Mayor de San Marcos; 2008.

3. Kassic F, Rabot S, Uhl M, Huber W, Min Qin H, Helma
C, et al. Chemoprotective effects of garden cress (Lepidium sativum) and its constituents towards 2-amino-3-methyl-imidazo[4,5-f]quinolone (IQ)induced genotoxic effects and colonic preneoplastic lesions. Carcinogenesis. 2002;23(7):1155-61.

4. Alzamora L, Colona E, Acero N, Galán A, MuñozMingarro D, Linares $F$ y col. Efecto citotóxico del extracto metanólico de tres ecotipos de Lepidium peruvianum Chacón sobre líneas celulares HeLa y HT-29. Rev Peru Biol. 2007;13(3):219-21.

5. Castaño M. Maca (Lepidium peruvianum Chacon): composición química y propiedades farmacológicas. Clin Cancer Res. 2006;12(5):1447-53.

6. Ganzera M, Zhao J, Muhammad I, Khan IA. Chemical profiling and standardization of Lepidium meyenii (maca) by reversed phase high performance liquid chromatography. Chem Pharmaceutical Bull. 2002;50(7):988-91

7. Gonzáles GF. Maca: de la tradición a la ciencia. Instituto de investigaciones de la altura. Universidad Peruana Cayetano Heredia. 2006.

8. Rubio J, Dang H, Gong M, Liu X, Chen S, Gonzales $\mathrm{G}$. Aqueous and hydroalcoholic extracts of Black Maca (Lepidium meyenii) improve scopolamineinduced memory impairment in mice. Food Chem Toxicol. 2007; 45:1882-90.

9. Zhang HY, Zheng CY, Yan H, Wang ZF, Tang LL, Gao X, Tang XC. Potential therapeutic targets of huperzine A for Alzheimer's disease and vascular dementia. Chem Biol Interact. 2008;175(1-3):396402.

10. Wang R, Tang XC. Neuroprotective effects of huperzine A. A natural cholinesterase inhibitor for the treatment of Alzheimer's disease. Neurosignals. 2005;14(1-2):71-82.

11. Giacobini E. Implicación de un nuevo enzima en la enfermedad de Alzheimer. J Neural Transm. 2002;109:1053-65.

12. Cruz R, Almaguer Meliam W, Bergado Rosado JA. [Glutathione in cognitive function and neurodegeneration]. Rev Neurol. 2003;36:877-86.

Manuscrito recibido el 15 de noviembre 2010 y aceptado para su publicación el 10 de enero 2011.

\section{Correspondencia:}

Dra. Raquel Oré Sifuentes

Centro de Investigación en Bioquímica y Nutrición, Facultad de Medicina, UNMSM

Av. Grau 755. Lima 1, Perú.

Correo electrónico: kelaore@yahoo.es. 